\title{
Broadband Dielectric Spectroscopy of Cell Cultures
}

\author{
Xiue Bao, Ilja Ocket, Member, IEEE, Juncheng Bao, Jordi Doijen, Ju Zheng, Dries Kil, Zhuangzhuang Liu, \\ Bob Puers, Fellow, IEEE, Dominique Schreurs, Fellow, IEEE, and Bart Nauwelaers, Senior Member, IEEE
}

\begin{abstract}
Broadband dielectric spectroscopy measurements of biological materials within $\mathrm{RF} /$ microwave range can reveal cellular information, which is of important value in biological and medical researches. Here we present a platform that combines a miniaturized coplanar waveguide (CPW) transmission line (TL) sensor and a special CPW fed interdigitated capacitor (IDC), which allows us to measure the complex permittivity of cell cultures from $300 \mathrm{kHz}$ to $50 \mathrm{GHz}$. The CPW-TL sensor and the CPW-IDC sensor are integrated with an SU-8 microfluidic channel, enabling measurements of microliter or even nano-liter volumes of liquids and suspensions. Due to the accurate alignment of the SU-8 polymer and the reliable lift-off fabrication procedure, we are able to minimize the measurement errors caused by the sensors' dimension tolerance. To ensure accurate complex permittivity extraction of the tested material, related calibrations and de-embedding processes are explained. With the measurement of deionized water as a validation, the platform is used to measure the complex permittivity of both a yeast cell culture and a mammalian cell culture. We elaborate on the interesting findings and discuss future possibilities.
\end{abstract}

Index Terms-Broadband, dielectric spectroscopy, mammalian cells, RF/microwave measurement, yeast cells.

\section{INTRODUCTION}

$\mathbf{E}$ LECTRICAL approaches, especially dielectric spectroscopy techniques that demonstrate the interaction between electromagnetic fields and materials, have been proven to be useful for a broad range of research purposes including biological research [1]-[3]. In recent decades, $\mathrm{RF} /$ microwave dielectric spectroscopy attracts increasing attention for non-invasive and non-destructive biological investigations [4]-[6]. Within $\mathrm{RF} /$ microwave frequency range, many significant properties of electromagnetic waves converge, which are suitable for biological research. First,

Manuscript received April XX, XXXX; revised August XX, XXXX.

This work was supported in part by the KU Leuven under projects $\mathrm{C} 2$ $\left(\mu^{2} \mathrm{BIO}\right)$, in part by the KU Leuven under project Hercules, and in part by the FWO. This paper is an expanded version from the IEEE MTT-S International Microwave Symposium (IMS2018), Philadelphia, PA, USA, June 10-15, 2018. (Corresponding author: Xiue Bao.)

X. Bao, J. Bao, D. Schreurs, and B. Nauwelaers are with the TELEMIC Division of Department of Electrical Engineering (ESAT), KU Leuven, 3001, Leuven, Belgium. e-mail: xiue.bao@esat.kuleuven.be (see https://www.esat.kuleuven.be/telemic/People-of-telemic/00106873).

I. Ocket is with the Div. ESAT-TELEMIC, KU Leuven, 3001, Leuven, Belgium, and also with the Interuniversity Microelectronics Center (IMEC), 3001, Heverlee, Belgium.

J. Doijen is with the Laboratory of Functional genomics \& proteomics, Zoological Institute, KU Leuven, Belgium, and also with the Laboratory of Virology and Chemotherapy, Rega Institute for Medical Research, KU Leuven, Belgium.

J. Zheng was with the Department of Biology, Functional Biology, KU Leuven, 3001, Heverlee, Belgium.

D. Kil and B. Puers are with the Div. ESAT-MICAS, KU Leuven, 3001, Leuven, Belgium.

Z. Liu is with the Material Department, KU Leuven, 3001, Leuven, Belgium. the wavelengths are comparable to the dimensions of the living matter and biological materials, resulting in complete penetration of these electromagnetic waves into the materials of interest [7]. Second, the dielectric properties that are highly related to biological parameters of the tested tissue and cells can be accurately translated into $\mathrm{RF} /$ microwave readouts. Analytical techniques of $\mathrm{RF} /$ microwave measurement results have been optimized and well-described. Finally, water, the largest constitution of any biological materials or living cells [8], shows its relaxation in RF/microwave frequency range (at around $20 \mathrm{GHz}$ ), due to its molecular reorientation dynamic. Therefore, the electromagnetic interaction with the living materials is assumed to be maximized in this frequency range.

One dominated application of the $\mathrm{RF} /$ microwave spectroscopy is tissue characterization with an open-ended coaxial method [9]. However, tissue measurements are currently not cost effective as they require large amounts of sample in every test. Moreover, tissue analysis provides limited information about the various consisting cells, which cannot meet the demands in biological and medical researches. For example, in the drug discovery process, to find an effective component for a certain disease, in vitro screening and mechanistical studies using cell-based assays play irreplaceable roles before performing preclinical assessments and clinical trials. Early research efforts [5], [10] demonstrate that various biological materials can present different dispersion properties at quite different frequencies. Obviously, the complicated composition of a single cell, a lipid bilayer encompassing an aqueous solution with various components and subcellular compartments [11], dynamic ion migrations through cell membrane [12], presence of highly ionic solution, etc., could potentially lead to particular and distinguishable dispersion properties within a broadband $\mathrm{RF} /$ microwave range. Therefore, it is necessary to observe the cells in a broadband frequency range in order to obtain detailed cellular information.

The advances of chip materials [13] and the rapid developments in MEMS fabrication technologies [14], [15] make it possible to characterize and analyze microliter or even nano-liter volumes of biological liquids and cell cultures in a non-invasive way. A representative example is the microfluidic structure, integration with various miniaturized designs resulting in hundreds of lab-on-chip devices [16] and micro total analysis systems ( $\mu \mathrm{TAS})$ [17]. When the dielectric spectroscopy technique is combined with microfluidic structures, it can benefit a lot the early biological analysis [18] and possibly in turn disease diagnoses as well. The potential, efficiency and simplicity of this combination has been previously demonstrated [8], [19]. One way is to use resonators to characterize the complex permittivity of a 
material under test (MUT), by building a linear mathematical model between the complex permittivity and the transmission loss variations and resonance frequency shifts [20]. However, it can only be used for very narrow bandwidth measurements, directly relating to the device self-resonance properties. Another attractive approach is to combine the microfluidic structure with a transmission line (TL), extracting the complex permittivity from the distributed parameters $C$ and $G$ or the effective complex permittivity of the TL [21], [22]. This method can provide broadband information, but it can only be used at high frequencies where the wavelengths are comparable to the TL's dimension, presenting ideal distributed characteristics.

In this work, aimed at obtaining the very broadband spectroscopy of cell cultures, a special coplanar waveguide (CPW) based interdigitated capacitor (IDC) is introduced, in addition to a CPW based TL sensor. The CPW fed IDC sensor shows a linear relationship between its equivalent circuit parameters and the MUT permittivity properties, which has been validated with cell concentration measurements [19] and dynamic measurements of yeast cells [23]. This paper is organized as follows: section II specifically explains the biological dispersion properties and measurement principles, especially the complex permittivity extraction protocol of the MUT; section III describes the fabrication techniques of the miniaturized sensors, the entire measurement setup, and the data processing procedure; in section IV, measurement results on yeast cell culture and mammalian cell culture are presented and analyzed; conclusions are drawn in the final section.

\section{THEORIES AND TOPOLOGY}

\section{A. Electrical Protocol of Biological Materials}

When the electromagnetic waves penetrate across a dielectric material, the material will be polarized depending on the applied frequency and the material's composition. According to Schwan's description [10], the polarization within RF/microwave range can be interpreted with four main dispersions: $\alpha$ dispersions that appear at low frequencies and are generally associated with the diffusion processes of ionic species, $\beta$ dispersions that are situated at megahertz range and are mainly due to the interfacial polarization across the cellular plasma membranes and their interactions with the intra- and extra-cellular electrolytes, $\gamma$ dispersions that are located in microwaves and mainly corresponds to the polarization of electrical dipole moments of aqueous and small biological molecules, and $\delta$ dispersions that appear between the $\beta$ and $\gamma$ dispersions [11] and are attributed to the dipole moments of large molecules, such as proteins, protein-bound water, biopolymers, and cellular organelles.

Havriliak and Negami [24] proposed a general relaxation expression to represent the various dispersion properties, as

$$
\varepsilon_{r}^{*}=\varepsilon_{\infty}+\sum_{n=1}^{N} \frac{\Delta \varepsilon_{n}}{\left(1+\left(j \omega \tau_{n}\right)^{1-\alpha_{n}}\right)^{\beta_{n}}}+\frac{\sigma_{0}}{j \omega \varepsilon_{0}}
$$

where $\varepsilon_{r}^{*}$ refers to the broadband complex permittivity, $\varepsilon_{\infty}$ the infinite high-frequency permittivity, $N$ the number of relaxation terms, $\Delta \varepsilon_{n}$ the $n$th dielectric intensity (when $N$

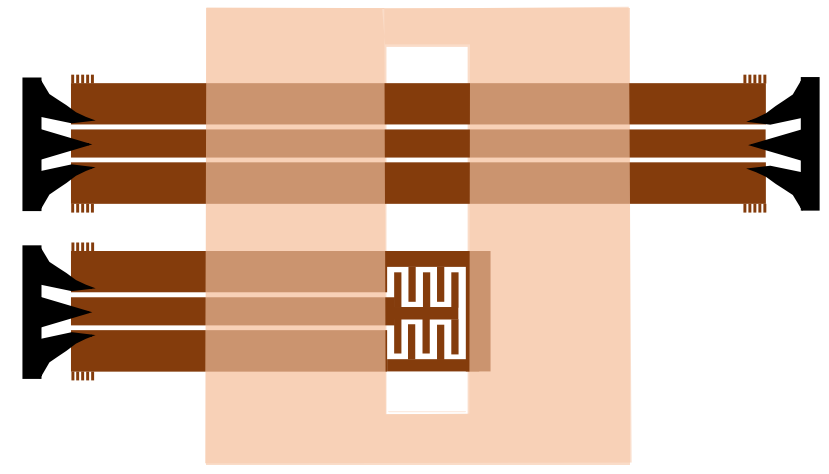

(a)

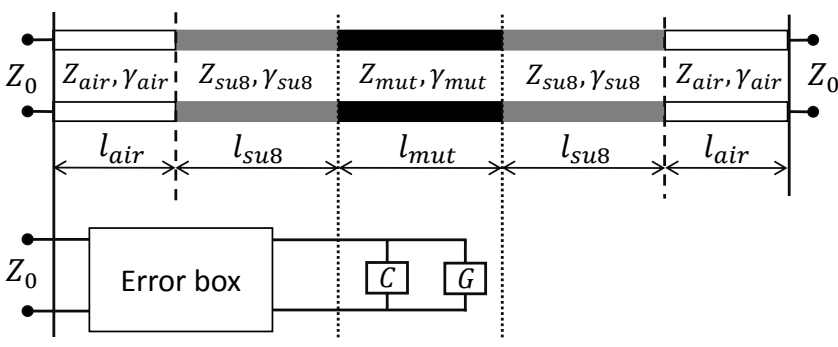

(b)

Fig. 1. (a) Structure schematics of the coplanar waveguide transmission line (CPW-TL) sensor and the CPW fed interdigitated capacitor (IDC) sensor. (b) Equivalent electrical circuits of the two sensors.

$=1, \Delta \varepsilon=\varepsilon_{s}-\varepsilon_{\infty}, \varepsilon_{s}$ the static permittivity), $j^{2}=-1, \omega$ the angular frequency, defined by $\omega=2 \pi f$ ( $f$ the frequency of the applied electromagnetic field), $\tau_{n}$ the relaxation time, $\alpha_{n}$ and $\beta_{n}$ the parameters that describe the spread of the relaxation time around its average value, $\sigma_{0}$ the static conductivity, $\varepsilon_{0}$ the permittivity of free space. Even though the equation is empirical, it is widely used to model dielectric spectra, and by adjusting the parameters $\alpha_{n}$ and $\beta_{n}$ in (1), it easily turns into the Cole-Davidson function $(\alpha=0,0<\beta<1)$ [25], the Cole-Cole function $(0<1-\alpha<1, \beta=1)$ [26], the Debye function $(\alpha=0, \beta=1)$ [27], etc.

\section{B. Data Treatment Protocol}

As previously discussed, the frequency dependent dispersions that are often expressed by the dielectric spectroscopy are distinct among different materials, which becomes the characterization principle in this study. Fig. 1(a) presents the structures of the proposed microfluidic CPW-TL and CPW-IDC, which are used for high frequency and low frequency measurements, respectively. The interdigitated electrodes of the CPW-IDC sensor were designed by the end of the central conductor of a CPW line. Fig. 1(b) depicts the equivalent circuits of the two sensors. For the CPW-TL sensing area, the wavelengths of the applied electric fields are comparable to its dimensions, so it is modeled as an infinite series of two-port elementary components. The circuit of each component consists of four distributed per unit length parameters - resistance $R_{u}$, inductance $L_{u}$, capacitance $C_{u}$, and conductance $G_{u}$. They relate to the complex propagation 
TABLE I

COEFFICIENT VALUES OBTAINED FROM SIMULATION WHEN $\varepsilon_{r}^{\prime \prime}$ IS SET CONSTANT

\begin{tabular}{|c|c|c|c|c|}
\hline$\varepsilon_{r}^{\prime \prime}$ & $a_{r r}\left(a_{R}\right)$ & $a_{i r}$ & $b_{R}$ & $b_{r r}$ \\
\hline 0 & $1.502 \times 10^{-14}$ & $3.297 \times 10^{-16}$ & $8.529 \times 10^{-14}$ & $8.529 \times 10^{-14}$ \\
\hline 20 & $1.525 \times 10^{-14}$ & $1.452 \times 10^{-16}$ & $6.729 \times 10^{-14}$ & $7.266 \times 10^{-14}$ \\
\hline 40 & $1.520 \times 10^{-14}$ & $-1.705 \times 10^{-17}$ & $6.241 \times 10^{-14}$ & $7.888 \times 10^{-14}$ \\
\hline & $\begin{array}{c}<a_{r r}>=1.516 \times 10^{-14} \\
\sigma_{a_{r r}}=9.823 \times 10^{-17}\end{array}$ & $\begin{array}{c}<a_{i r}>=1.526 \times 10^{-16} \\
\sigma_{a_{i r}}=1.417 \times 10^{-16}\end{array}$ & $\begin{array}{c}<b_{R}>=7.166 \times 10^{-14} \\
\sigma_{b_{R}}=9.840 \times 10^{-15}\end{array}$ & $\begin{array}{c}<b_{r r}>=7.894 \times 10^{-14} \\
\sigma_{b_{r r}}=5.156 \times 10^{-15}\end{array}$ \\
\hline
\end{tabular}

TABLE II

COEFFICIENT VALUES OBTAINED FROM SIMULATION WHEN $\varepsilon_{r}^{\prime}$ IS SET CONSTANT

\begin{tabular}{|c|c|c|c|c|}
\hline$\varepsilon_{r}^{\prime}$ & $a_{r i}$ & $a_{i i}\left(a_{I}\right)$ & $b_{I}$ & $b_{i i}$ \\
\hline 1 & $-7.274 \times 10^{-16}$ & $1.555 \times 10^{-14}$ & $1.482 \times 10^{-14}$ & $1.449 \times 10^{-14}$ \\
\hline 40 & $-2.686 \times 10^{-16}$ & $1.565 \times 10^{-14}$ & $1.226 \times 10^{-14}$ & $6.449 \times 10^{-15}$ \\
\hline 80 & $-4.118 \times 10^{-16}$ & $1.547 \times 10^{-14}$ & $2.832 \times 10^{-14}$ & $2.969 \times 10^{-14}$ \\
\hline & $\begin{array}{c}<a_{r i}>=-4.692 \times 10^{-16} \\
\sigma_{a_{r i}}=1.916 \times 10^{-16}\end{array}$ & $\begin{array}{c}<a_{i i}>=1.555 \times 10^{-14} \\
\sigma_{a_{i i}}=7.157 \times 10^{-17}\end{array}$ & $\begin{array}{c}<b_{I}>=1.847 \times 10^{-14} \\
\sigma_{b_{I}}=7.047 \times 10^{-15}\end{array}$ & $\begin{array}{c}<b_{i i}>=1.688 \times 10^{-14} \\
\sigma_{b_{i i}}=9.636 \times 10^{-15}\end{array}$ \\
\hline
\end{tabular}

constant $\gamma$ and characteristic impedance $Z_{c}$ of the complete sensing region by

$$
\begin{gathered}
\gamma=\sqrt{\left(R_{u}+j \omega L_{u}\right)\left(G_{u}+j \omega C_{u}\right)} \\
Z_{c}=\sqrt{\frac{R_{u}+j \omega L_{u}}{G_{u}+j \omega C_{u}}}
\end{gathered}
$$

where $\gamma$ and $Z_{c}$ can be readily calculated from the ABCD matrix that is directly transformed from the scattering (S-)parameters of the MUT loaded transmission line [28]. It has been verified both by a conformal mapping technique [29] and a two-dimensional (2D) finite element method (FEM) [30] that at every frequency point the distributed $C_{u}$ and $G_{u}$ are linearly dependent on the complex permittivity of the material loaded on top of the CPW electrodes.

In terms of the CPW-IDC sensing area, as its dimensions are much smaller than the wavelengths of the electromagnetic fields transmitted in the device, the electric fields are position-independent at the sensing area. Therefore, the IDC sensing area is modeled as a lumped equivalent circuit [19] as shown in Fig. 1(b), where the equivalent $C$ and $G$ relate to the S-parameters $S_{m u t}$ of the MUT covered sensing area by

$$
G+j \omega C=\frac{1}{Z_{0}} \cdot \frac{1-S_{m u t}}{1+S_{m u t}}
$$

The values of $C$ and $G$ are associated to the substrate and the MUT on both sides of the IDC fingers. To mathematically demonstrate this association, a 3D FEM simulation is performed on the IDC sensing area where a given MUT $\left(\varepsilon_{r}^{*}=\varepsilon_{r}^{\prime}-j \varepsilon_{r}^{\prime \prime}\right)$ is loaded. At three different constant MUT imaginary permittivities $\left(\varepsilon_{r}^{\prime \prime}=0,20,40\right)$, the MUT real permittivity $\varepsilon_{r}^{\prime}$ is set at $1,5,10, \ldots, 80$ separately and the related simulated S-parameters are recorded. Similarly, at three different constant MUT real permittivities $\left(\varepsilon_{r}^{\prime}=1,40,80\right)$, the MUT imaginary permittivity $\varepsilon_{r}^{\prime \prime}$ increases from 0 to 40 in a step size of 2.5 and the related simulation results are recorded. From the recorded S-parameters, the equivalent $C$ and $G$ values under different MUT settings are separately calculated with (4) [19].

If we assume that the MUT complex permittivity has the following mathematical relationship with the equivalent $C$ and $G$ of the IDC sensing area,

$$
\left(\begin{array}{c}
C \\
G / \omega
\end{array}\right)=\left(\begin{array}{ll}
a_{r r} & a_{r i} \\
a_{i r} & a_{i i}
\end{array}\right) \cdot\left(\begin{array}{c}
\varepsilon_{r}^{\prime} \\
\varepsilon_{r}^{\prime \prime}
\end{array}\right)+\left(\begin{array}{c}
b_{r r} \\
b_{i i}
\end{array}\right)
$$

where six coefficients are required to be quantificationally determined: $a_{r r}$ and $a_{r i}$ represent the impacts of $\varepsilon_{r}^{\prime}$ and $\varepsilon_{r}^{\prime \prime}$ on the $C$ value, respectively; whereas $a_{i r}$ and $a_{i i}$ quantify the impacts of $\varepsilon_{r}^{\prime}$ and $\varepsilon_{r}^{\prime \prime}$ on $G / \omega$, respectively; $b_{r r}$ and $b_{i i}$ are related to the bare IDC sensing area. Calculation results of the six coefficients are reported in Table I and Table II, together with their respective mean values and standard deviations. From the obtained data, especially from the fact that the ratios $a_{r r} / a_{i r}$ and $a_{i i} / a_{r i}$ are both at around 100 , we can straightforwardly arrive at the conclusion that compared to $a_{r r}$ and $a_{i i}, a_{r i}$ and $a_{i r}$ are neglectable in (5). The small standard deviations of $a_{r r}$ and $a_{i i}$ can also verify that the cross dependence is neglectable. Therefore, the following two independent linear equations between the MUT complex permittivity and the equivalent $C$ and $G / \omega$ of the IDC sensor are obtained

$$
\begin{gathered}
C=a_{R} \cdot \varepsilon_{r}^{\prime}+b_{R} \\
G / \omega=a_{I} \cdot \varepsilon_{r}^{\prime \prime}+b_{I}
\end{gathered}
$$

Fitting the 3D simulation results with the two equations, we find that $a_{R}$ has the same value as $a_{r r}$, and $a_{I}$ is the same as $a_{i i}$. Furthermore, $b_{R}$ and $b_{I}$ are comparable to $b_{r r}$ and $b_{i i}$, respectively, which can be readily deduced from the fitting results in Table I and Table II.

Taking a closer look at the values $a_{r r}\left(a_{R}\right)$ and $a_{i i}\left(a_{I}\right)$ in Table I and Table II, we can assume that they are the same, 
and consequently, by introducing the loss $\operatorname{tangent} \tan \delta$, the following formula is obtained

$$
\tan \delta=\frac{\varepsilon_{r}^{\prime \prime}}{\varepsilon_{r}^{\prime}}=\frac{\Delta G}{\omega \cdot \Delta C}
$$

The linearity in (8), which has been tested at frequencies from $300 \mathrm{kHz}$ to $1 \mathrm{GHz}$ on a similar IDC [23], allows to extract the complex permittivity of the dielectric materials under investigation from the measured $\mathrm{RF} /$ microwave S-parameters.

\section{FABRICATION AND MEASUREMENT}

\section{A. Sensor Fabrication}

The two microfluidic CPW based characterization sensors were patterned on a $1 \mathrm{~mm}$ thick $10.16 \mathrm{~cm}$ diameter fused silica (quartz) wafer, due to its isotropic permittivity and low loss property. The widths of center conductor, gaps, and ground planes of the CPW structure were designed at $110 \mu \mathrm{m}, 20 \mu \mathrm{m}$, and $150 \mu \mathrm{m}$, respectively. The microfluidic channel widths of the two sensor were both designed at $500 \mu \mathrm{m}$. There were 22 fingers at the CPW-IDC sensing area, with the finger length, width, gap being $130 \mu \mathrm{m}$, $20 \mu \mathrm{m}$, and $20 \mu \mathrm{m}$, respectively. The feeding segment of the CPW-IDC sensor or on either side of the microfluidic CPW-TL sensor was composed of a $1.5 \mathrm{~mm}$ long quartz/air region and a $4.5 \mathrm{~mm}$ long quartz/SU-8 region. To accurately calibrate the two microfluidic sensors, several additional multiline thru-reflect-line (TRL) calibration standards [31] and a de-embedding standard were co-fabricated on the same wafer as the sensors, which was aimed at minimizing the variability in the properties and dimensions of the CPW electrodes due to fabrication tolerance. All of the standards were designed to have the same cross-section dimension as the CPW sensors. (a) Microelectrodes

i. Photoresist coating

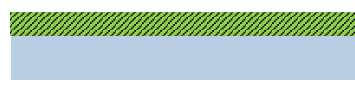

ii. Photoresist patterning $\mathscr{W} \mathscr{W} \mathscr{W} \mathscr{Z} \mathscr{Z}$

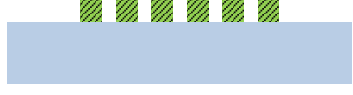

iii. $\mathrm{Cr} / \mathrm{Au}$ sputtering

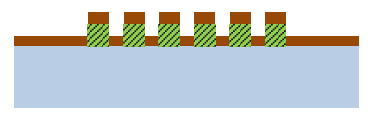

iv. Removal of photoresist

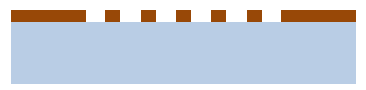

(b) Microfluidics

i. Polymer coating

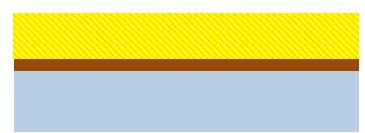

ii. Polymer patterning UV
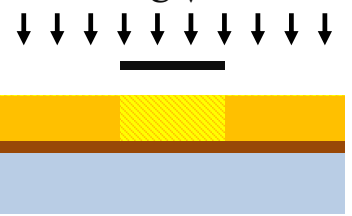

iii. Channel development

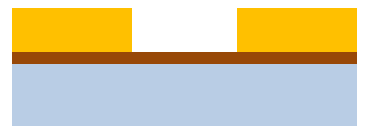

Fig. 2. The fabrication procedures on (a) metalization process of the microelectrodes and (b) lithography process of the SU-8 microfluidic layer.
The de-embedding standard was designed to have exactly the same dimensions as the feeding segment, i.e., a $6 \mathrm{~mm}$ long bare CPW line loaded with $4.5 \mathrm{~mm}$ long SU-8 polymer.

Fig. 2 depicts the fabrication prototype of the devices and calibration standards. After the cleaning of a quartz wafer, the positive photoresist S1818 was spin-coated on the wafer and patterned with the traditional UV photolithography. Then, the metallization was done through the sputter deposition technique: a $\sim 160 \mathrm{~nm}$ thick chromium (Cr) film, working as the adhesion layer to guarantee the adhesion between gold and quartz, followed by a $\sim 500 \mathrm{~nm}$ thick gold layer. As shown in Fig. 2(a), the sputtering process was followed by a lift-off process, eliminating the photoresist with the top metal into a solvent solution.

Next, the SU-8 microfluidic channel, used for assuring liquid confinement, was fabricated perpendicularly to the CPW central conductor using the technical strategy presented in Fig. 2(b). With the main advantage of being biocompatible, simply molded, requiring low fabricating cost, and of high aspect ratio features, SU-8 can provide good alignment when integrated with microstructures and microdevices [13]. The height of the SU-8 microfluidic channel is $400 \mu \mathrm{m}$, and consequently, the liquid volume within the microfluidic channel is $0.09 \mu \mathrm{L}$ (i.e., $0.45 \times 0.5 \times 0.4 \mathrm{~mm}^{3}$ ). In addition, a mould was fabricated with a $3 \mathrm{D}$ printing technique [32] to enable the replication of a disposable polydimethylsiloxane (PDMS) layer, which will be covered on top of the microfluidic channel during every liquid measurement. The SU-8 channel height has been designed larger than the sum of the CPW center conductor and two gaps widths $(110 \mu \mathrm{m}+2 \times 20 \mu \mathrm{m})$ [29], so that the electromagnetic fields primarily interact with fluids contained within the SU-8 channels rather than the top air or PDMS.

\section{B. Measurement Platform}

All measurements were performed on a probe station that was equipped with two movable microwave 40A-GSG-150C picoprobes, as shown in Fig. 3(a). For a broadband dielectric spectroscopy characterization, the probes were connected to a Keysight M9375A PXIe module for low frequency measurements and an Agilent E8361A vector network analyzer (VNA) for high frequency measurements, using the CPW-IDC and the CPW-TL (as shown in Fig. 3(b) and (c)), respectively. During each measurement, a plexiglass spacer was placed under the wafer to remove the parasitic coupling between the gold conductor and the holding metal chuck. Moreover, thermal equilibrium was considered when performing all measurements and calibrations. An air conditioning system was used to keep the laboratory temperature constant, and a temperature control system (Temptronic TPO3210B) equipped on the probe station was used to help the devices and liquids under test reach the desired temperature set-point.

The raw complex S-parameters as a function of frequency were recorded. In high frequency on-wafer measurements, we acquired 1601 frequency points from $10 \mathrm{MHz}$ to $50 \mathrm{GHz}$ on a linear frequency scale. For low frequency measurements, the frequency ran from $300 \mathrm{kHz}$ to $5 \mathrm{GHz}$ and 801 points 

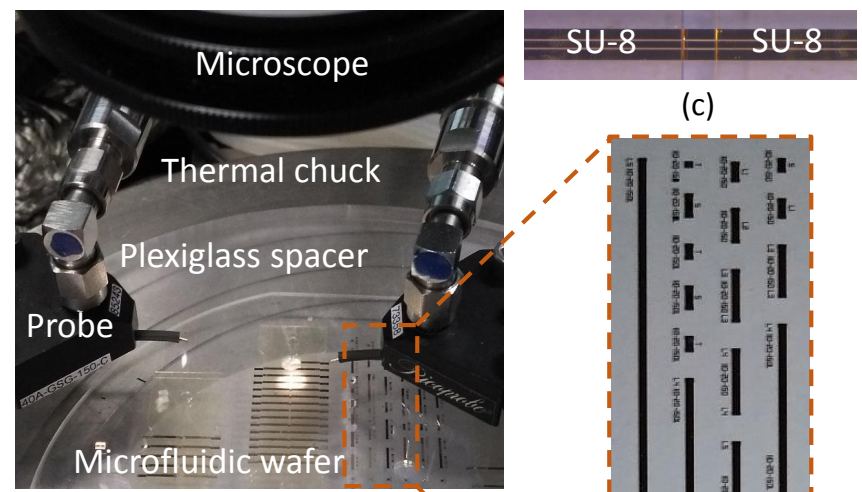

(c)

(a)

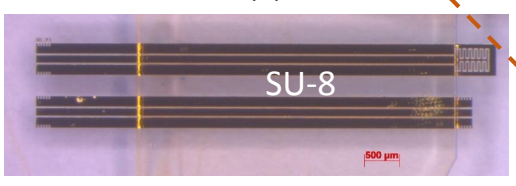

(b)

(d)

Fig. 3. The measuring system and associated standards: (a) the probe station setup; (b) the microfluidic CPW-IDC sensor and the de-embedding standard; (c) zoom-in view of the microfluidic CPW-TL sensor; (d) the multiline TRL calibration standards.

on a $\log$ frequency scale were recorded. The intermediate frequency (IF) bandwidths of both high and low frequency range were set at $20 \mathrm{~Hz}$, and the power of each VNA was set at $-20 \mathrm{dBm}$ to avoid any unwanted microwave heating effects. The temperature was set at $20^{\circ} \mathrm{C}$ for all measurements in this study. After measuring the empty devices and reference liquids, the cell cultures were injected into the microfluidic channel with a micropipette, after which the microfluidic channel was covered with a PDMS sheet for about one minute to let the liquid become still.

\section{On-chip Calibration}

Choosing an appropriate calibration technique is essential and mandatory in accurate RF/microwave measurements. In this study, a two-tier calibration strategy is used to remove the errors and parasitic parameters between VNAs and MUT reference planes. Among a large number of first-tier on-chip calibration techniques, the most commonly used methods are the Short-Open-Load-Thru (SOLT) calibration and the multiline TRL calibration [31]. The SOLT calibration, a well-established method for VNA calibration, assumes the load symmetrical and of identical impedance value to the measurement system impedance [33], which is reasonable at low frequencies. However, to our experience, at high frequencies, the load definition is frequency dependent and affected by the probe mounting and placement, meaning that the SOLT calibration method is not recommended for high frequency measurements. The Multiline TRL calibration has been considered the benchmark for other on-wafer calibration methods because it makes the least assumptions about the calibration standards compared to other calibration methods. However, it occupies much expensive wafer space when used for low frequency measurements. Therefore, in this study, we adopt SOLT and multiline TRL calibration methods for low frequency and high frequency measurements, respectively, to move the reference planes from VNAs to the probe-tips. The calibration process from $10 \mathrm{MHz}$ to $50 \mathrm{GHz}$ was performed with the multiline TRL standards lithographically fabricated on the quartz wafer (shown in Fig. 3(d)). The lengths of the multiline TRL calibration standards were $0.42 \mathrm{~mm}$ (thru), $0.21 \mathrm{~mm}$ (short), $1.27 \mathrm{~mm}$ (line 1), $3.22 \mathrm{~mm}$ (line 2), $5.933 \mathrm{~mm}$ (line 3), $10.5 \mathrm{~mm}$ (line 4), and $25 \mathrm{~mm}$ (line 5), respectively. The SOLT calibration method used for $300 \mathrm{kHz}$ to $5 \mathrm{GHz}$ was achieved by a commercial alumina CPW CS- 5 impedance standards substrate from GGB Industries and its associated parameters provided by the vendor.

Next, to move the reference planes from the probe-tips to the sensing area of the sensor, a second-tier calibration (i.e., de-embedding) is required. The de-embedding principle of the CPW-TL sensor is presented in Fig. 1(b), where the complete structure can be expressed by the following matrix cascading [28], as

$$
T_{\text {tot }}=T_{\text {air } L} \cdot T_{\text {su } 8 L} \cdot T_{\text {mut }} \cdot T_{\text {su } 8 R} \cdot T_{\text {air } R}
$$

where $T_{\text {airL } L} / T_{\text {airR } R}, T_{s u 8 L} / T_{\text {su } 8 R}, T_{m u t}$ are the cascade matrices of the left/right bare CPW feeding line, left/right SU-8 loaded CPW feeding line, and the MUT loaded sensing area, respectively. $T_{t o t}$ is the cascade matrix of the entire structure between the two probe tips. The bare feeding part and the SU-8 feeding part are assumed to be two identical transmission lines, and thus can be expressed with four distributed parameters $R_{u}, L_{u}, C_{u}, G_{u}$. The four parameters of the bare line can be readily calculated from the frequency dependent propagation constant obtained through the multiline TRL calibration process. By measuring the empty CPW-TL sensor or the de-embedding line shown in Fig. 3(b), assuming $R_{u}$ and $L_{u}$ are the same as that of bare line [28], [30], we readily achieve $C_{u}$ and $G_{u}$ of the SU-8 line. With these frequency dependent values, $T_{a i r L}, T_{a i r R}, T_{s u 8 L}$, and $T_{s u 8 R}$ can be calculated. Consequently, we are able to move the reference planes to the sensing area, and yield the matrix $T_{m u t}$ of the MUT loaded area by matrix operation of (9).

At low frequencies, the feeding section of the IDC sensor is considered as an error box (as shown in Fig. 1(b)). To remove the error box, we can directly measure the additional de-embedding line (Fig. 3(b)) and calibrate it with the two-port SOLT calibration technique. The calibrated S-parameters are assumed to be the same as that of the IDC sensor's feeding part, and therefore, the de-embedding process [28] is expressed as [19]

$$
S_{m u t}=\frac{S_{t o t}-S_{f e e d 11}}{S_{f e e d 12} S_{f e e d 21}-S_{f e e d 11} S_{f e e d 22}+S_{t o t} S_{f e e d 22}}
$$

where $S_{t o t}, S_{m u t}$, and $S_{\text {feed }}$ are the calibrated complex S-matrices of the complete CPW-IDC structure up to the probe-tip, the sensing area loaded with MUT, and the CPW feeding region, respectively.

\section{Measurement Results and Discussion}

\section{A. Measurement of Yeast Cell Culture}

The broadband dielectric spectroscopy measurement was first carried out on the BY 4741 yeast cell strain [23]. We 


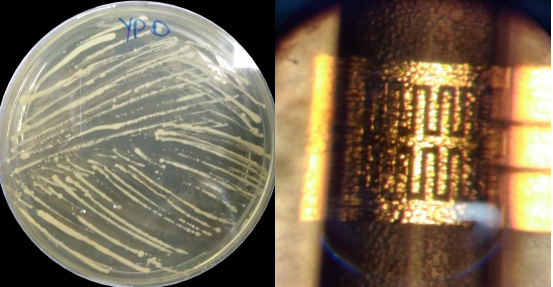

(a)

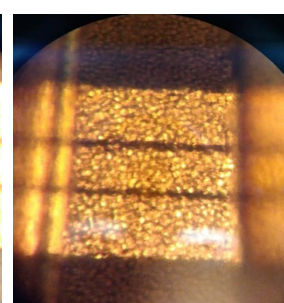

(c)
Fig. 4. Photos of (a) agar plate with cultured BY 4741 yeast cells, and the distribution of the U-87 MG mammalian cell culture on (b) the CPW-IDC sensor and (c) the CPW-TL sensor.

prepared the BY 4741 cell samples in the following way: they were first streaked on a $9 \mathrm{~cm}$ diameter plastic Petri dish that had been sterilized and filled with solidified YPD agar media; the plate was then inverted and put in an incubator,

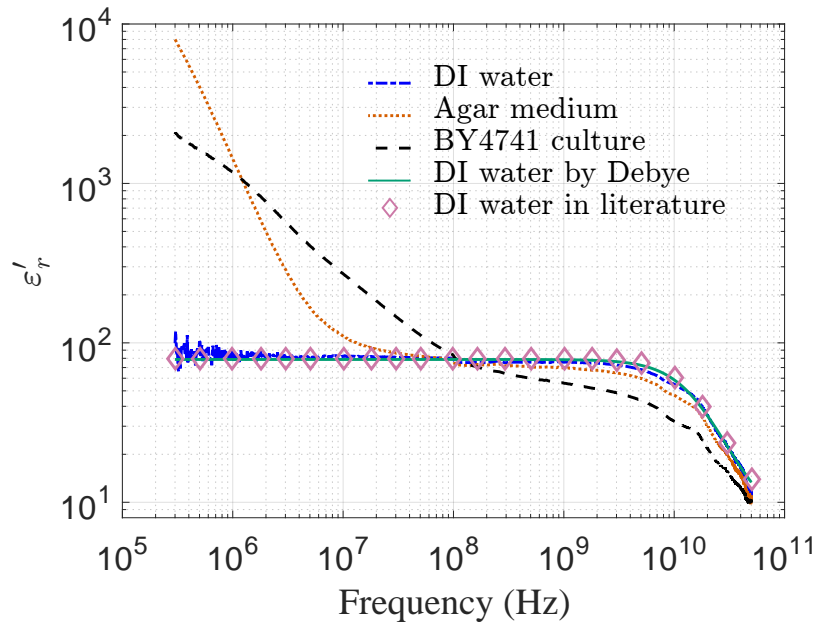

(a)

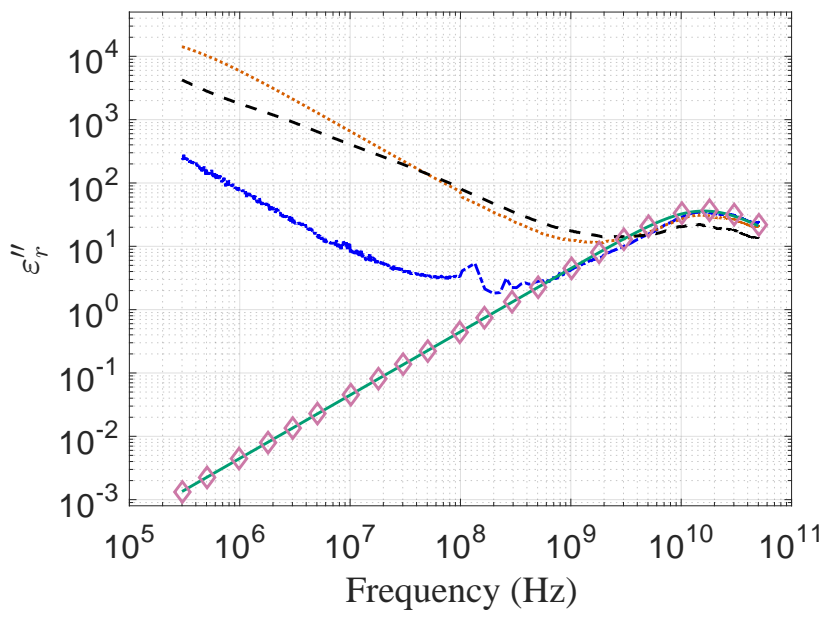

(b)

Fig. 5. Broadband dielectric spectroscopy measurements ((a) real and (b) imaginary permittivity) of BY 4741 cell culture at $20^{\circ} \mathrm{C}$ within the frequency range from $300 \mathrm{kHz}$ to $50 \mathrm{GHz}$. The permittivity properties of deionized water [27] and the solid agar medium are also presented, working as a reference. The scale is in the decimal logarithm base. incubating the cells for about 48 hours at $30^{\circ} \mathrm{C}$ (as shown in Fig. 4(a)); next, one yeast cell colony on the incubated plate, which was grown from one single BY 4741 cell, was picked up with a sterilized pipette tip for the spectroscopy measurement. Working as validation measurements, the S-parameters of the two sensors before and after loading deionized (DI) water were recorded. In addition, as a control, the clean YPD agar medium that was obtained from the same culture plate as where the BY 4741 yeast cells had grown was measured. Six groups of DI water measurements were repeated before and after the medium and cell culture measurements. The repeatability error of DI water's permittivity is about $1.7 \%$, which indicates the measurement uncertainty of the proposed platform. All measurements were carried out at $20^{\circ} \mathrm{C}$ to avoid any effects caused by temperature difference.

The permittivity measurement results with the frequency ranging from $300 \mathrm{kHz}$ to $50 \mathrm{GHz}$ are all presented in Fig. 5, together with data from literature on DI water [27]. To make the curves more readable, data on both horizontal (frequency)

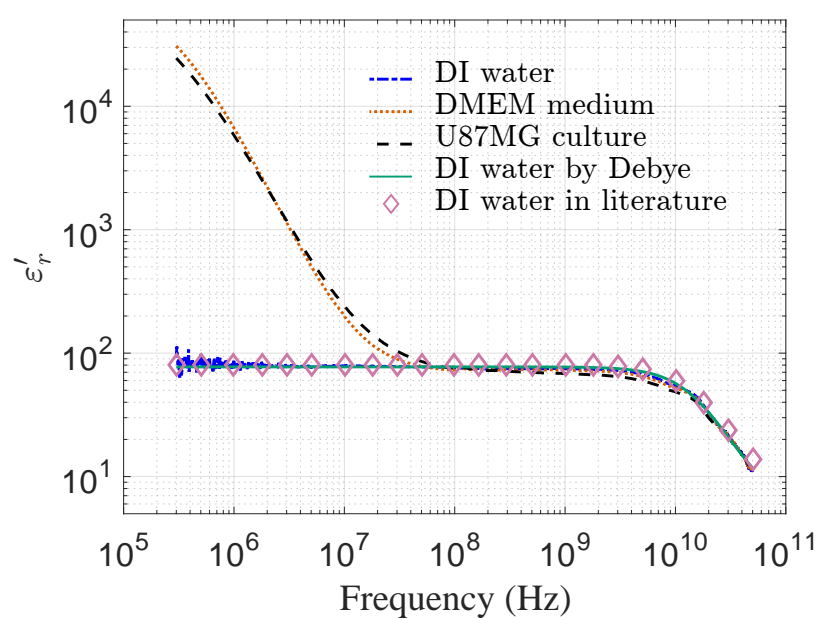

(a)

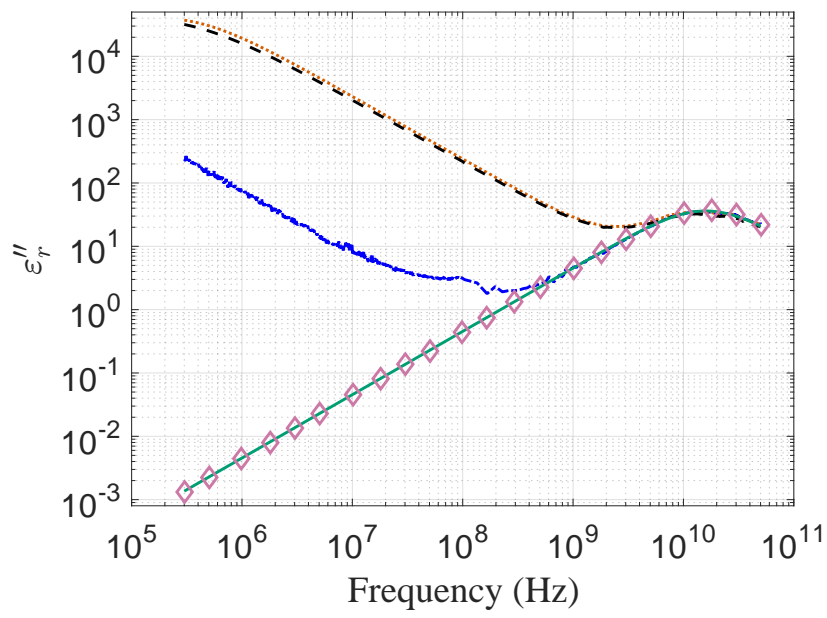

(b)

Fig. 6. Broadband dielectric spectroscopy measurements ((a) real and (b) imaginary permittivity) of U-87 MG mammalian cell culture at $20^{\circ} \mathrm{C}$ within the frequency range from $300 \mathrm{kHz}$ to $50 \mathrm{GHz}$. The permittivities of deionized water [27] and the pure culture medium are also presented, working as a reference. The scale is in the decimal logarithm base. 
and vertical axes (measured real/imaginary permittivity) are shown on the log scale. For further analysis, the extracted complex permittivity of DI water is fitted to the Debye formula of (1), where $N=1, \alpha=0$, and $\beta=1$. The fitted parameters $\varepsilon_{\infty}, \varepsilon_{s}$, and $\tau$ of DI water are $6.09,80.17$, and 9.6, respectively. The data calculated with the fitted parameters are also presented in Fig. 5. Notably, both the measured real permittivity of the DI water and the real part result calculated using the fitted Debye parameters show strong consistency with literature data [27]. Even though the measured imaginary permittivity of DI water shows good agreement with literature for frequencies higher than 300 $\mathrm{MHz}$, there is an almost linear decrease (with the slope at around -1) when increasing the frequency from 300 $\mathrm{kHz}$ to approximate $300 \mathrm{MHz}$. Interestingly, the imaginary permittivity calculated with the fitted Debye parameters still show good agreement with literature values. The constant real permittivity and the unexpected high effective imaginary permittivity at low frequencies might indicate that there are extremely few ions in the DI water during the preparation with the Milli- ${ }^{\circledR}$ Water Purification System.

\section{B. Measurement of Mammalian Cell Culture}

The broadband complex permittivity measurement was also carried out on U-87 MG mammalian cell culture from the American Type Culture Collection (Rockville, MD, USA). The U-87 MG cells were cultured in T75 flasks (Falcon ${ }^{\circledR}$, Thermofisher Scientific), in the complete growth medium DMEM (Dulbecco's Modified Eagle Medium, Gibco ${ }^{\circledR}$, ThermoFisher Scientific) supplemented with $10 \%$ Fetal Bovine Serum (Gibco ${ }^{\circledR}$, ThermoFisher Scientific) at $37^{\circ} \mathrm{C}$ in a humidified incubator with 5\% CO2. Cells were trypsinated using $0.25 \%$ Trypsin/EDTA (Gibco ${ }^{\circledR}$, Thermofisher Scientic) and resuspended in fresh growth medium prior to seeding them in the measurement device. The approximate conductivity of this growth medium is $\sigma_{0}=1.4 \mathrm{~S} / \mathrm{m}$ [34]. To reduce contamination, all devices were cleaned using Milli- ${ }^{\circledR}$ water, dried and UV sterilized before the cells were seeded. There were in average 6 million U-87 MG cells per milliliter culture medium. As the measured volume within the microfluidic channel was $0.09 \mu \mathrm{L}$, there were about 540 cells on the sensing area during measurements. The photos of Fig. 4(b) and (c) present the distributions of the U-87 MG cells on the two devices, which were observed under the probe station microscope.

Fig. 6 shows both real and imaginary parts of the extracted relative permittivity of the tested mammalian cell culture at frequencies from $300 \mathrm{kHz}$ up to $50 \mathrm{GHz}$. Similar to the yeast cell culture characterization, the pure liquid DMEM without cells and the DI water were also measured, with the measurement results also being presented in Fig. 6. The measured DI water permittivities were fitted to Debye formula, and the fitted results together with the literature data [27] are also presented in Fig. 6 as a reference. For the measurement of DI water, we can draw similar conclusions as with the yeast cell culture.

\section{Analysis and Discussion}

Further observation of Fig. 5 and Fig. 6 results in some preliminary conclusions. First, the imaginary part permittivities of agar medium, BY 4741 culture, DMEM medium, and U-87 MG culture show similar trends to the measured DI water, i.e., with the frequency increasing from $300 \mathrm{kHz}$ to about $3 \mathrm{GHz}$, their imaginary permittivities decrease approximately linearly on the log-log scale, whereas at frequencies above $3 \mathrm{GHz}$, we see a typical relaxation similar to DI water, presenting symmetric relaxation peaks at approximate $20 \mathrm{GHz}$. Second, interestingly, at low frequencies, agar's $\varepsilon_{r}^{\prime \prime}$ is decreasing with almost the same speed as DI water, with the slope at around -1 , whereas BY 4741 culture's $\varepsilon_{r}^{\prime \prime}$ decreases slower. Though less pronounced, similar phenomenon is observed on DMEM medium and U-87 MG culture. The -1 slope of imaginary permittivity on a log-log scale indicates that both agar medium and DMEM medium are typical conductive fluids. Third, the real permittivities of the four media/cultures behave similar to that of DI water at

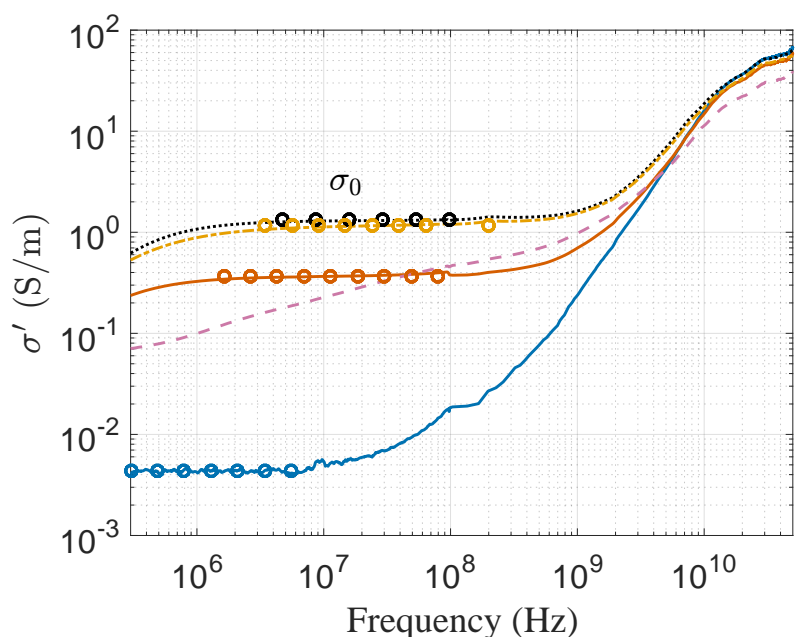

(a)

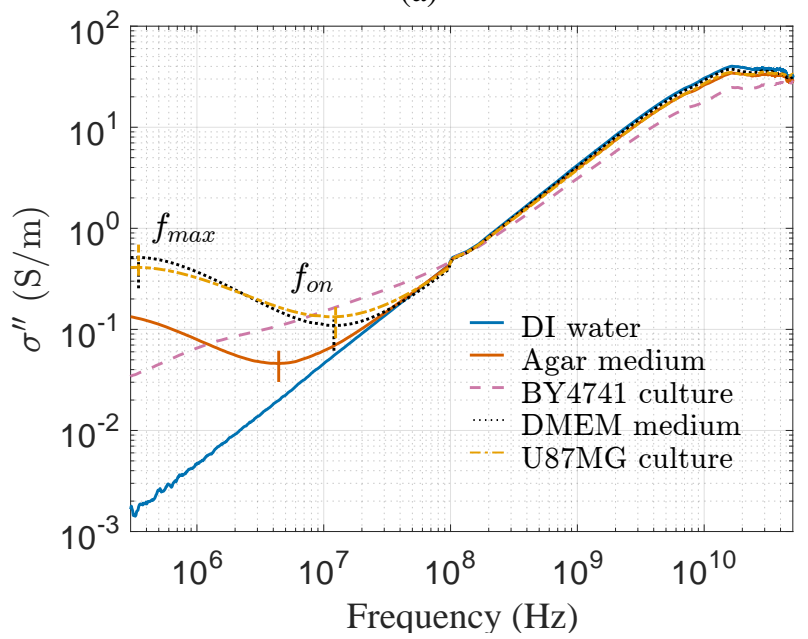

(b)

Fig. 7. The calculated frequency dependent complex conductivity of the agar medium, the DMEM medium, the BY 4741 yeast cell culture, and the U-87 MG mammalian cell culture with the frequency sweeping from $300 \mathrm{kHz}$ to 50 $\mathrm{GHz}$. The complex conductivity of deionized water is presented as a reference. 
frequencies between about $100 \mathrm{MHz}$ and $50 \mathrm{GHz}$. Finally, the large difference of the real permittivities occurs in the frequency range from $300 \mathrm{kHz}$ to $100 \mathrm{MHz}$, where the measured $\varepsilon_{r}^{\prime}$ values of the four MUTs are not constant but much larger than that of DI water. The large values are dominated by the electrode polarization (EP) [35], [36] due to the high ion concentration in the MUTs. Notably, for the yeast and the mammalian cell culture results, the poorly conducting cells can shield part of the electrodes from the ions, thus reducing the EP effects compared to the pure ionic culturing medium.

The EP phenomenon is determined by details of both the electrodes and the material under observation [37], and especially highly related to the ionic conductivity of the material. The higher the ionic conductivity of the culture medium or the cell suspension, the greater the EP effect, which, in some cases, may completely obscure the dielectric behavior. Using the real and imaginary permittivity results with the Kramers-Kronig transforms is an effective way to analyze the conductivity, but the presence of EP makes the process complicated. An alternative is the introduction of complex conductivity $\sigma^{*}=\sigma^{\prime}+j \sigma^{\prime \prime}$, which can be directly converted from the dielectric complex permittivity with $\sigma^{*}=$ $j \omega \varepsilon_{0} *\left(\varepsilon_{r}^{\prime}-j \varepsilon_{r}^{\prime \prime}\right)$ [36]. The calculated complex conductivity of the four measured MUTs are presented in Fig. 7.

The lowest points marked by $f_{\text {on }}$ in Fig. 7(b) are the onset of the EP phenomenon, whereas the points marked with $f_{\max }$ present full developments of the EP. The EP effect mainly appears at low frequencies, and $f_{\text {on }}$ indicates the upper boundary of its dominant range. The EP onset frequency points of agar medium, DMEM medium, and U-87 MG cell culture are $4.40 \mathrm{MHz}, 11.630 \mathrm{MHz}$, and 11.773 $\mathrm{MHz}$, respectively, which are in good consistency with the phenomenon presented on the real permittivities in Fig. 5 and Fig. 6. It is clear that the $f_{\text {on }}$ of agar medium is much lower than other liquids, which might be related with its semisolid-state properties. The constant values marked with small circles on the curves in Fig. 7(a) represent their respective estimated static conductivities [36]. The observed static conductivity $\sigma_{0}$ of agar medium, DMEM medium [34], and U-87 MG cell culture are $0.3666 \mathrm{~S} / \mathrm{m}, 1.3396 \mathrm{~S} / \mathrm{m}$, and $1.1873 \mathrm{~S} / \mathrm{m}$, respectively. Clearly shown in Fig. 7, BY 4741 cell culture presents the most interesting measurement results: neither constant $\sigma_{0}$ nor $f_{\text {on }}$ and $f_{\max }$ can be obtained readily from the plots.

The EP effect can be modeled as an effective capacitance in parallel with an effective conductance, which are in series with the MUT's equivalent capacitance and conductance [38]. According to the dielectric spectroscopy curves of the four MUTs in Fig. 5 and Fig. 6, the EP effects can be modeled by a constant phase element (CPE) [37], [39], and dielectric properties of the bulk MUT can be modeled with a ubiquitous Cole-Cole function [38]. The relative complex permittivity of the MUT can be directly extracted by fitting the corresponding measured permittivity data at frequencies higher than $f_{\text {on }}$ to (1) where $N=1,0<1-\alpha<1$, and $\beta=1$ (i.e., the Cole-Cole function). The previously fitted Debye parameters $\varepsilon_{\infty}, \varepsilon_{s}$, and $\tau$ of DI water and the previously estimated $\sigma_{0}$ of the four
MUTs (used agar's estimated $\sigma_{0}$ for BY 4741 cell culture) work as the initial values. The fitted dispersion parameters of agar medium, BY 4741 cell culture, DMEM medium, and U-87 MG cell culture are presented in Table III. Obviously, the fitted $\sigma_{0}$ results in Table III are comparable to those estimated from Fig. 7. As expected, the dispersion parameters of BY 4741 cell culture in Table III are clearly distinct from the respective parameters of other liquids. This might indicate that there is at least one dominant dispersion occurring within the lower RF and microwave frequency range, which is obscured by the EP effects. Further research is needed in order to help clarify these findings.

TABLE III

THE PARAMETERS THAT DESCRIBE THE DISPERSION PROPERTIES OF THE PURE MEDIUM AND CELL CULTURES

\begin{tabular}{cccccc}
\hline MUT & $\varepsilon_{\infty}$ & $\varepsilon_{s}$ & $\tau$ & $\alpha$ & $\sigma_{0}$ \\
\hline Agar & 2.1440 & 67.5791 & 9.5017 & 0.0644 & 0.3480 \\
BY4741 & 0.6466 & 57.9321 & 12.6072 & 0.2139 & 0.4602 \\
DMEM & 1.7202 & 74.0212 & 9.4900 & 0.0545 & 1.3379 \\
U87MG & 1.7538 & 70.3309 & 9.7411 & 0.0840 & 1.1806 \\
\hline
\end{tabular}

\section{CONCLUSION}

Aiming at observing the multiple dispersions occurring in the small biological materials, we presented the interaction of electromagnetic waves and two types of cells within the broadband frequency from $300 \mathrm{kHz}$ to $50 \mathrm{GHz}$. The broadband measurements were carried out with a characterization platform that combines a $\mathrm{CPW}$ transmission line sensor and a one-port CPW interdigitated capacitor based sensor. The two sensors are integrated with a microfluidic structure and fabricated on a $1 \mathrm{~mm}$ thick $10.16 \mathrm{~cm}$ diameter quartz wafer, so that the position of the material under test can be precisely determined. By involving reliable calibration protocols and de-embedding algorithms, we quantitatively determined the complex permittivities of agar medium, BY 4741 cells that are cultured within agar medium, DMEM medium, and of the U-87 MG mammalian cell culture.

\section{ACKNOWLEDGMENT}

The authors would like to thank Dr. V. Franssens from the Functional Biology and Prof. W. Luyten from the Zoological Institute for productive discussion on cell properties and measurements, Prof. D. Schols from the laboratory of Virology and Chemotherapy at Rega Institute for providing the mammalian U87-MG cell culture, FabLab Leuven for the PDMS mold fabrication, Leuven NanoCentre for device fabrication, and KU Leuven Central Electronic Service (CDE) for technical support.

\section{REFERENCES}

[1] E. C. Gregg and K. D. Steidley, "Electrical counting and sizing of mammalian cells in suspension," Biophys. J., vol. 5, no. 4, pp. 393-405, Jul. 1965.

[2] I. Giaever and C. R. Keese, "Micromotion of mammalian cells measured electrically," Proc. Natl. Acad. Sci. U.S.A., vol. 88, no. 17, pp. 7896-7900, Sep. 1991. 
[3] R. D. Stoy, K. R. Foster, and H. P. Schwan, "Dielectric properties of mammalian tissues from 0.1 to $100 \mathrm{MHz}$ : a summary of recent data," Physics in Medicine \& Biology, vol. 27, no. 4, pp. 501-513, Apr. 1982.

[4] J. Wegener, C. R. Keese, and I. Giaever, "Electric cell-substrate impedance sensing (ECIS) as a noninvasive means to monitor the kinetics of cell spreading to artificial surfaces," Experimental cell research, vol. 259, no. 1, pp. 158-166, Aug. 2000.

[5] F. Kremer, "Dielectric spectroscopy-yesterday, today and tomorrow," $J$. Non-Cryst Solids, vol. 305, no. 1, pp. 1-9, Jul. 2002.

[6] C. Gabriel, "Compilation of the dielectric properties of body tissues at RF and microwave frequencies," King's Coll London (United Kingdom) Dept. of Physics, Tech. Rep., 1996

[7] A. Shaw et al., "Rapid analysis of high-dimensional bioprocesses using multivariate spectroscopies and advanced chemometrics," in Bioanalysis and Biosensors for Bioprocess Monitoring. Springer, 1999, pp. 83-113.

[8] K. Grenier et al., "Recent advances in microwave-based dielectric spectroscopy at the cellular level for cancer investigations," IEEE Trans. Microw. Theory Techn., vol. 61, no. 5, pp. 2023-2030, Apr. 2013.

[9] D. Xu, L. Liu, and Z. Jiang, "Measurement of the dielectric properties of biological substances using an improved open-ended coaxial line resonator method," IEEE Trans. Microw. Theory Techn., vol. 35, no. 12, pp. 1424-1428, Dec. 1987.

[10] H. P. Schwan, "Electrical properties of tissue and cell suspensions," in Adv. Biol. Med. Phys. Elsevier, Dec. 1957, vol. 5, pp. 147-209.

[11] A. Oleinikova, P. Sasisanker, and H. Weingärtner, "What can really be learned from dielectric spectroscopy of protein solutions? a case study of ribonuclease a," J. Phys. Chem. B, vol. 108, no. 24, pp. 8467-8474 May 2004

[12] A. Schwab, V. Nechyporuk-Zloy, A. Fabian, and C. Stock, "Cells move when ions and water flow," Pflügers Archiv-European Journal of Physiology, vol. 453, no. 4, pp. 421-432, Jan. 2007.

[13] J. Liu et al., "Process research of high aspect ratio microstructure using SU-8 resist," Microsys. Technol, vol. 10, no. 4, pp. 265-268, May 2004.

[14] C. Liu, "Recent developments in polymer MEMS," Adv. Mater, vol. 19, no. 22, pp. 3783-3790, Oct. 2007.

[15] E. Verpoorte and N. De Rooij, "Microfluidics meets MEMS," Proc. IEEE, vol. 91, no. 6, pp. 930-953, Jun. 2003.

[16] P. Abgrall and A. Gue, "Lab-on-chip technologies: making a microfluidic network and coupling it into a complete microsystema review," $J$. Micromech. Microeng, vol. 17, no. 5, pp. R15-R49, 2007.

[17] T. Vilkner, D. Janasek, and A. Manz, "Micro total analysis systems. recent developments," Anal. Chem., vol. 76, no. 12, pp. 3373-3386, Apr. 2004

[18] Y. Yang et al., "Distinguishing the viability of a single yeast cell with an ultra-sensitive radio frequency sensor," Lab Chip, vol. 10, no. 5, pp. 553-555, Jan. 2010

[19] X. Bao et al., "A planar one-port microwave microfluidic sensor for microliter liquids characterization," IEEE J-ERM, vol. 2, no. 1, pp. 10-17, Feb. 2018

[20] T. Chretiennot, D. Dubuc, and K. Grenier, "A microwave and microfluidic planar resonator for efficient and accurate complex permittivity characterization of aqueous solutions," IEEE Trans. Microw. Theory Techn., vol. 61, no. 2, pp. 972-978, Dec. 2013.

[21] G. Facer, D. Notterman, and L. Sohn, "Dielectric spectroscopy for bioanalysis: From $40 \mathrm{hz}$ to $26.5 \mathrm{GHz}$ in a microfabricated wave guide," Appl. Phys. Lett., vol. 78, no. 7, pp. 996-998, Feb. 2001.

[22] S. Liu, I. Ocket, M. Cauwe, D. Schreurs, and B. Nauwelaers, "Sensitivity analysis of broadband on-wafer dielectric spectroscopy of yeast cell suspensions up to $110 \mathrm{GHz}$," IEEE Microw. Wirel. Compon. Lett., vol. 25, no. 3, pp. 199-201, Mar. 2015.

[23] X. Bao et al., "Yeast cell growth monitoring using microwave measurements correlated to optical absorbance," in 2018 IEEE MTT-S Int. Microwave Symp. Dig. Philadelphia, PA, 2018, pp. 1-3.

[24] S. Havriliak and S. Negami, "A complex plane representation of dielectric and mechanical relaxation processes in some polymers," Polymer, vol. 8, pp. 161-210, 1967.

[25] D. W. Davidson and R. H. Cole, "Dielectric relaxation in glycerol, propylene glycol, and n-propanol," J. Chem. Phys., vol. 19, no. 12, pp. 1484-1490, Dec. 1951.

[26] K. S. Cole and R. H. Cole, "Dispersion and absorption in dielectrics i. alternating current characteristics," J. Chem. Phys., vol. 9, no. 4, pp. 341-351, 1941.

[27] W. J. Ellison, "Permittivity of pure water, at standard atmospheric pressure, over the frequency range $0-25 \mathrm{THz}$ and the temperature range $0-100{ }^{\circ}$ C," J. Phys. Chem. Ref. Data, vol. 36, no. 1, pp. 1-18, Mar. 2007.
[28] R. B. Marks and D. F. Williams, "A general waveguide circuit theory," Journal of Research-National Institute of Standards and Technology, vol. 97, pp. 533-533, Sep.-Oct. 1992.

[29] X. Bao et al., "Coplanar waveguide for dielectric material measurements at frequencies from $140 \mathrm{GHz}$ to $220 \mathrm{GHz}$," in 90th ARFTG Conference Digest-Fall. Boulder, CO, USA, 28 Nov.-1 Dec. 2017, pp. 1-4.

[30] J. C. Booth, N. D. Orloff, J. Mateu, M. Janezic, M. Rinehart, and J. A. Beall, "Quantitative permittivity measurements of nanoliter liquid volumes in microfluidic channels to $40 \mathrm{GHz}$," IEEE Trans. Instrum. Meas., vol. 59, no. 12, pp. 3279-3288, Dec. 2010

[31] R. B. Marks, "A multiline method of network analyzer calibration," IEEE Trans. Microw. Theory Techn., vol. 39, no. 7, pp. 1205-1215, Jul. 1991.

[32] F. Rengier et al., "3D printing based on imaging data: review of medical applications," Int. J. Comput. Assist. Radiol. Surg., vol. 5, no. 4, pp. 335-341, Jul. 2010

[33] P. Kirby, L. Dunleavy, and T. Weller, "Load models for cpw and microstrip solt standards on gaas," in ARFTG Conference Digest-Fall, 56th, vol. 38. IEEE, 2000, pp. 1-11.

[34] W. M. Arnold and G. Fuhr, "Increasing the permittivity and conductivity of cellular electromanipulation media," in 1994 Industry Applications Society Annual Meeting, vol. 2. Denver, CO, USA, 1994, pp. 1470-1476

[35] F. Bordi, C. Cametti, and T. Gili, "Reduction of the contribution of electrode polarization effects in the radiowave dielectric measurements of highly conductive biological cell suspensions," Bioelectrochemistry, vol. 54, no. 1, pp. 53-61, Aug. 2001.

[36] A. Serghei, M. Tress, J. Sangoro, and F. Kremer, "Electrode polarization and charge transport at solid interfaces," Phys. Rev. B, vol. 80, no. 18, p. 184301 , Nov. 2009.

[37] T. Pajkossy, "Impedance of rough capacitive electrodes," J. Electroanal. Chem., vol. 364, no. 1-2, pp. 111-125, Jan. 1994.

[38] X. Bao, S. Liu, I. Ocket, Z. Liu, D. Schreurs, and B. Nauwelaers, "A modeling procedure of the broadband dielectric spectroscopy for ionic liquids," IEEE Trans. Nanobiosci., Accepted, 2018.

[39] J. R. Macdonald, "Note on the parameterization of the constant-phase admittance element," Solid State Ion., vol. 13, no. 2, pp. 147-149, May 1984.

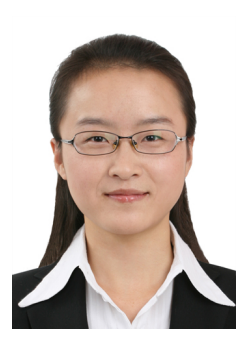

Xiue Bao (S'17) was born in Baoding, China. She received the B.Sc. degree in information engineering in 2011, and the M.Sc. degree in metallurgical engineering in 2013, in the University of Science and Technology Beijing, Beijing, China. She is currently pursuing the $\mathrm{Ph} . \mathrm{D}$. degree in electrical engineering at the University of Leuven, Leuven, Belgium. Her current research interests focus on microwave biomedical applications, including broadband dielectric spectroscopy; biosensor design; microwave-microfluidic structure design and fabrication; RF, microwave, and millimeter wave measurement and calibration. She was one of the recipient of the IEEE Microwave Theory and Techniques Society Graduate Fellowship in 2018

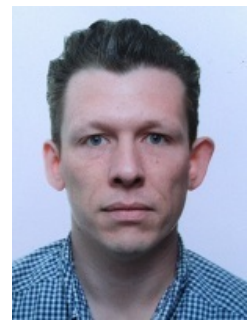

Ilja Ocket (M'09) received the M.Sc. and Ph.D. degrees in electrical engineering from KU Leuven, Leuven, Belgium, in 1998 and 2009, respectively. $\mathrm{He}$ has been with imec, Leuven, and KU Leuven, since 1999. With imec, he is currently involved in millimeter-wave antenna modules and packaging for 79- and 140-GHz radar. With KU Leuven, he leads the group working in the area of microwave and millimeter-wave applications for biology and medicine, and is also involved in integrated couplers for (sub)millimeter plastic waveguides. 


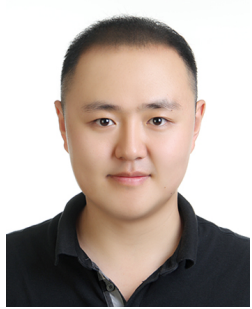

Juncheng Bao (S'17) received the M.Sc. degree in electrical engineering from KU Leuven, Leuven, Belgium, in 2013. He had an internship with imec, Leuven, in 2013. He is currently pursuing the Ph.D. degree in electrical engineering at KU Leuven, Leuven, Belgium. His current research interests include microwave biomedical applications, novel microwave-microfluidic devices design and fabrication, microwave and millimeter wave metrology.

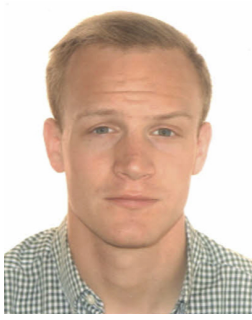

Jordi Doijen received his M.Sc degree in Biochemistry and biotechnology from KU Leuven, Belgium, in 2015. He is currently doing his $\mathrm{PhD}$ at the Zoological institute at KU Leuven under supervision of Prof. Liliane Schoofs. He studies the potential of cellular electrical impedance measurements for G-coupled-receptor-based drug discovery. This research is funded by the FWO (Flemish Research Funds, SB/ 151105). His research interests include drug discovery, receptor pharmacology, cell biology and cancer research.

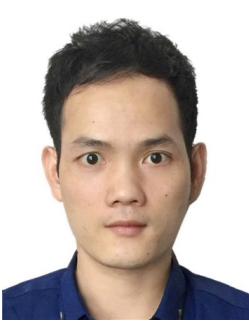

Ju Zheng received his M.Sc. degree from Harbin Institute of Technology (HIT, China) in 2013 and $\mathrm{Ph} . \mathrm{D}$. degree in Biology from K.U. Leuven. During his $\mathrm{PhD}$ at K.U. Leuven, he worked in the laboratory of Functional Biology, under supervisor of Prof. Joris Winderickx, investigated neurodegenerative diseases proteins in yeast model. $\mathrm{He}$ is now a PostDoc researcher at Southern University of Science and Technology (SUSTC, China).

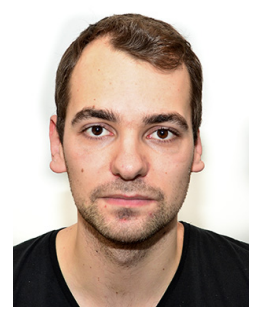

Dries Kil received the degree of M.S. of Nanoscience \& Nanotechnology from the Katholieke Universiteit Leuven, Belgium, in 2014. The subject of his M.S. thesis was the design and fabrication of a novel neurotrophic electrode based on controlled growth factor release. Since October 2014 he is working as a research assistant at the MICAS group, under the guidance of Prof. Robert Puers, obtaining a Ph.D in Electrical Engineering. His main research is focused on the development of flexible neural probes with a strong focus on biocompatibility and tissue integration.

Zhuangzhuang Liu Biography is not available now.

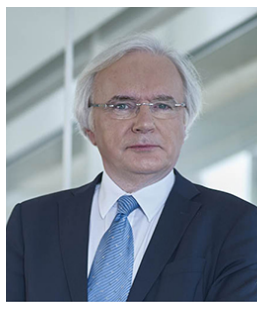

Robert Puers (M'86-SM'95-F'11) received the Ph.D. degree from Katholieke Universiteit Leuven (K.U. Leuven), Leuven, Belgium, in 1986. At K.U Leuven, he became Director of the Clean Room Facilities for Silicon and Hybrid Circuit Technology at the ESAT-MICAS laboratories. He was a European pioneer in the research on micromachining, MEMS and packaging techniques, mainly for biomedical implantable systems. To this purpose, he assembled the requested infrastructure, and installed a clean room in 1984, that now runs for more than 25 years under his guidance. Recently, microfluidic and optical MEMS based on polymers are forming the backbone of his sensor research. Beside MEMS, his work focuses also on low power systems, smart interfaces, inductive power and wireless communication. He published design guidelines to improve the efficiency of power induction in two books. He took major efforts to increase the impact of MEMS and microsystems in both the international research community as well as in industry. He helped to launch two spin-off companies.

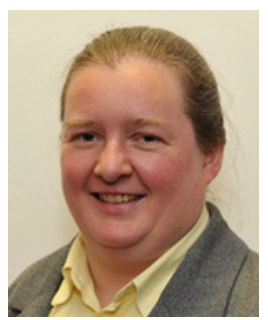

Dominique Schreurs (S'90-M'97-SM'02-F'12) received the M.Sc. degree in electronic engineering and $\mathrm{Ph} . \mathrm{D}$. degree from the University of Leuven, Leuven, Belgium. She is now a Full Professor with $\mathrm{KU}$ Leuven and Chair of LICT at KU Leuven. She has been a Visiting Scientist with Agilent Technologies, Santa Rosa, CA, USA; ETH Zurich, Zurich, Switzerland; and the National Institute of Standards and Technology, Boulder, CO, USA. Her current research interests include the microwave and millimeter-wave characterization and modeling of active devices and bioliquids, as well as the system design for wireless communications and biomedical applications.

Prof. Schreurs was an IEEE MTT-S Distinguished Microwave Lecturer and the Editor-in-Chief of the IEEE TRANSACTIONS ON MICROWAVE THEORY AND TECHNIQUES. She was the General Chair of the 2007, 2012, and 2018 ARFTG Conference. She was the Co-Chair of the European Microwave Conference in 2008. She is currently President-Elect of the IEEE MTT-S. She serves as the President of the ARFTG organization.

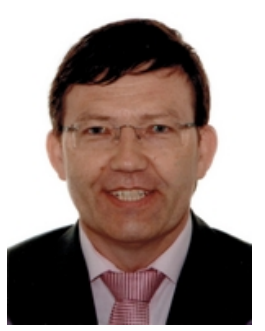

Bart Nauwelaers (S'80-M'86-SM'99) received the $\mathrm{Ph} . \mathrm{D}$. degree in Electrical Engineering from the KU Leuven in 1988. He also received the Master's degree in Design of Telecommunication Systems from Telecom ParisTech, Paris, France. Since 1981, he has been with the Department of Electrical Engineering, KU Leuven, where he has been involved in research on microwave antennas, passive components, interconnects, microwave integrated circuits and MMICs, linear and nonlinear device modeling, MEMS, and wireless communications.

Prof. Nauwelaers is the former chair of IEEE AP/MTT-Benelux and past chair of URSI-Benelux. 